\title{
Decreased angiogenesis and arthritic disease in rabbits treated with an $\alpha \mathbf{v} \beta 3$ antagonist
}

\author{
Chris M. Storgard, ${ }^{1}$ Dwayne G. Stupack, ${ }^{1}$ Alfred Jonczyk, ${ }^{2}$ Simon L. Goodman, ${ }^{3}$ \\ Robert I. Fox, ${ }^{4}$ and David A. Cheresh ${ }^{1}$ \\ ${ }^{1}$ Departments of Immunology and Vascular Biology (IMM24), The Scripps Research Institute, La Jolla, California 92037, USA
${ }^{2}$ Department of Medicinal Chemistry, and
${ }^{3}$ Department of Immunology and Oncology, Merck KGaA, 64271 Darmstadt, Germany
${ }^{4}$ Department of Rheumatology, Scripps Clinic, La Jolla, California 92037, USA \\ Address correspondence to: David A. Cheresh, Departments of Immunology and Vascular Biology (IMM24), The Scripps Research \\ Institute, 10550 N. Torrey Pines Road, La Jolla, California 92037, USA. Phone: (619) 784-8281; Fax: (619) 784-8926; \\ E-mail: cheresh@scripps.edu
}

Chris M. Storgard and Dwayne G. Stupack contributed equally to this work.

Received for publication April 17, 1998, and accepted in revised form October 21, 1998.

\begin{abstract}
Rheumatoid arthritis (RA) is an inflammatory disease associated with intense angiogenesis and vascular expression of integrin $\alpha \mathrm{v} \beta 3$. Intra-articular administration of a cyclic peptide antagonist of integrin $\alpha v \beta 3$ to rabbits with antigen-induced arthritis early in disease resulted in inhibition of synovial angiogenesis and reduced synovial cell infiltrate, pannus formation, and cartilage erosions. These effects were not associated with lymphopenia or impairment of leukocyte function. Furthermore, when administered in chronic, preexisting disease, the $\alpha v \beta 3$ antagonist effectively diminished arthritis severity and was associated with a quantitative increase in apoptosis of the angiogenic blood vessels. Therefore, angiogenesis appears to be a central factor in the initiation and persistence of arthritic disease, and antagonists of integrin $\alpha v \beta 3$ may represent a novel therapeutic strategy for RA.
\end{abstract}

J. Clin. Invest. 103:47-54 (1999).

\section{Introduction}

The rheumatoid arthritic joint is characterized by massive synovial proliferation and changes in synovial architecture resulting in interdigitating folds of tissue,termed pannus. The formation of active inflamed pannus is thought to be central to erosive disease and resulting joint destruction (1). Angiogenesis, the formation of new blood vessels, is one of the earliest histopathologic findings in rheumatoid arthritis (RA) and appears to be required for pannus development (2). This neovascularization is thought not only to maintain the chronic architectural changes via delivery of required blood-borne elements to the pannus but also to play an active role in inflammation as a source of both cytokine and protease activity (3). The expanded vascularbed volume resulting from angiogenesis may provide increased access for inflammatory cells to infiltrate the synovium (4). Although the factors specifically promoting angiogenesis in RA have not been identified, both synovial tissue and fluid are enriched in angiogenesis-promoting molecules. These include cytokines, such as basic fibroblast growth factor (bFGF) (5), interleukin-8, and vascular endothelial growth factor, and soluble adhesion molecules, such as vascular cell adhesion molecule and Eselectin (6). Interestingly, many of the available treatments for RA have been shown to possess some degree of antiangiogenic activity (7-9). In fact, treatments that suppress the angiogenic process may favorably impact disease course, as suggested by studies in an adjuvant-induced model of arthritis (10).

We have demonstrated previously that integrin $\alpha v \beta 3$ is both a marker and crucial effector for blood vessels undergoing angiogenesis $(11,12)$. Blockade of this integrin by either antibody or peptide antagonists induces apoptosis of angiogenic blood vessels in cytokine and tumor models of angiogenesis (11-13). In agreement with previous immunohistochemical studies $(14,15)$, we found that synovial blood vessels from RA patients show increased expression of integrin $\alpha v \beta 3$. These observations prompted the current study, in which treatment directed against vascular integrin $\alpha v \beta 3$ was assessed for its impact on arthritic disease in a rabbit model of RA.

In this report, we demonstrate that intra-articular administration of a cyclic peptide antagonist of $\alpha v \beta 3$ in bFGF-augmented antigen-induced arthritis (AIA) was associated with a quantitative increase in vascular apoptosis leading to the inhibition of synovial angiogenesis, as well as a reduction in joint swelling, synovial infiltrate, and pannus formation, in both early and well-established arthritis. Importantly, the $\alpha v \beta 3$ antagonist provided significant protection against the development of cartilage erosions. These results substantiate the development of $\alpha v \beta 3$ antagonists for future clinical trials in RA.

\section{Methods}

Materials. Ovalbumin (OVA), Freund's complete and incomplete adjuvants, and bovine type II collagen were from Sigma Chemical Co. (St. Louis, Missouri, USA). SDS (Bio-Rad Life Science Research, Hercules, California, USA), sodium chromate (Amersham Life Sciences, Arlington Heights, Illinois, USA), and Percoll (Pharmacia Biotech, Uppsala, Sweden) were purchased from suppliers as indicated. Goat anti-human von Willebrand factor (vWf) affinity-purified antibody (Enzyme Research Laboratories, South Bend, Indiana, USA) was used as a blood ves- 

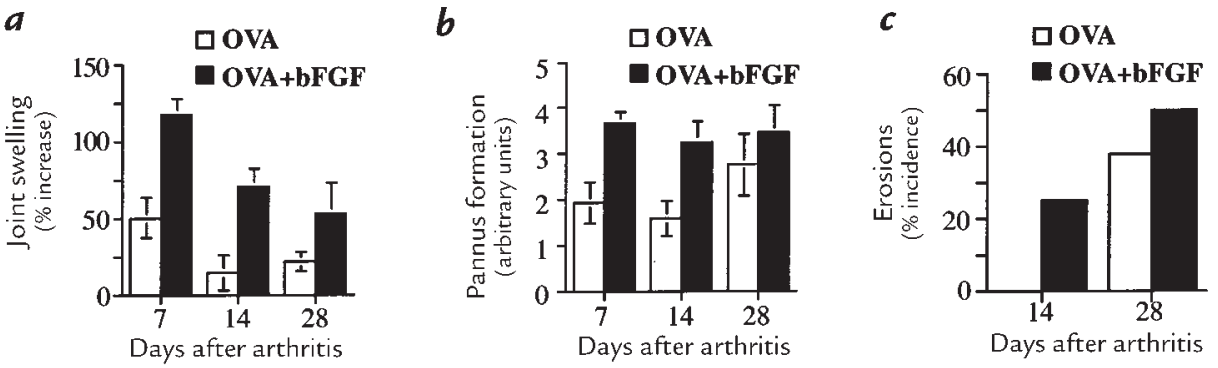

Figure 1

The proangiogenic cytokine bFGF intensifies arthritis. $(\boldsymbol{a})$ The addition of bFGF during induction of AIA enhanced arthritis severity compared with OVA alone, with increased joint swelling, greater pannus formation $(\boldsymbol{b})$, and earlier and more frequent erosive disease $(\boldsymbol{c})$. Pannus development was graded on a relative scale $0-5(0=$ normal to $5=$ macroscopic cartilage erosion $)$ (ref. 23). All data are expressed as mean $\pm \operatorname{SE}(n=8)$. AlA, antigeninduced arthritis; $b F G F$, basic fibroblast growth factor; OVA, ovalbumin.

sel marker. Monoclonal antibody (MAB) LM609 directed against integrin $\alpha v \beta 3$ (16) was produced and purified from the hybridoma. Fluorescein-conjugated, affinity-purified donkey anti-goat IgG and rhodamine-conjugated, affinity-purified donkey anti-mouse IgG were obtained from Jackson ImmunoResearch Laboratories (West Grove, Pennsylvania, USA). Recombinant bFGF was graciously supplied by J. Abraham (Scios Inc., Mountain View, California, USA). Cyclic peptides (17), cyclic Arg-Gly-Asp-D-Phe-Val (EMD 66203), cyclic Arg-Gly-Asp-D-Phe-[N-methyl]Val (EMD 85189), cyclic Arg- $\beta$-Ala-Asp-D-Phe-Val (EMD 69601), and cyclic Arg-GlyAsp-D-Phe-Lys[fluoresceincarboxylic acid] (EMD 80838) were graciously provided by Merck KGaA (Darmstadt, Germany) and reconstituted in $0.9 \%$ saline $(2 \mathrm{mg} / \mathrm{ml})$ adjusted to $\mathrm{pH} 7.4$ and stored at $-20^{\circ} \mathrm{C}$ until use.

Establishment of AIA. All experiments were performed in strict accordance with the guidelines of The Scripps Research Institute Department of Animal Resources. Arthritis was induced as described previously (18). Briefly, male New Zealand White rabbits $(2 \mathrm{~kg}$; Western Oregon Rabbit Co., Philomath, Oregon, USA) were immunized in multiple subcutaneous sites with a total of $1 \mathrm{ml}$ OVA $(20 \mathrm{mg} / \mathrm{ml})$ in Freund's complete adjuvant and boosted 2 weeks later with $0.6 \mathrm{ml} \mathrm{OVA}(20 \mathrm{mg} / \mathrm{ml})$ in Freund's incomplete adjuvant. Arthritis was induced 1 week later by bilateral knee intra-articular injection of $0.5 \mathrm{ml} \mathrm{OVA} / \mathrm{PBS}(20$ $\mathrm{mg} / \mathrm{ml})$ with or without recombinant bFGF $(6 \mu \mathrm{g} / \mathrm{ml})$. Animals were immobilized by predosing with $2 \mathrm{mg}$ acepromazine, followed by inhalation of $2.5 \%$ halothane $1.01 \mathrm{~min}^{-1}$ nitrous oxide/1.0 $1 \mathrm{~min}^{-1}$ oxygen anesthesia. Analgesia was provided with buprenorphine hydrochloride (Reckitt and Colman Pharmaceuticals Inc., Richmond, Virginia, USA), $0.2 \mathrm{ml}$ twice daily.

Treatment protocol. OVA-immunized rabbits were randomized, based on ELISA-determined OVA-antibody titers (see ref. 22), into groups treated with the $\alpha v \beta 3$ antagonist peptide (EMD 66203) or control peptide (EMD 69601) before arthritis induction with OVA/bFGF. Beginning $24 \mathrm{~h}$ after arthritis induction, and again on days 7,14 , and 21 , animals were anesthetized and peptides administered by intra-articular injection $(0.5 \mathrm{ml} / \mathrm{knee}, 2 \mathrm{mg} / \mathrm{ml})$. Rabbits were harvested on day 28 . To assess the effect of $\alpha v \beta 3$ antagonist treatment on chronic disease, rabbits with AIA were randomized (based on day 1 and day 7 knee diameter) into a control group (no treatment) or treatment with the $\alpha v \beta 3$ antagonist $(0.5 \mathrm{ml} / \mathrm{knee}, 2$ $\mathrm{mg} / \mathrm{ml}$ administered on days $14,16,18,21$, and 25).

Assessment of arthritis. Joint swelling, defined as increase in extended knee diameter from normal, was measured with dial calipers (0.254 mm; Fisher Scientific, Tustin, California, USA). All assessments were performed by a blinded observer. Immunofluorescence was performed as described previously (12) on cryosections of the infrapatellar fat-pad $(5 \mu \mathrm{m})$, using anti-vWf as a marker of blood vessels and MAB LM609 as a marker for integrin $\alpha v \beta 3$. Rhodamine-conjugated donkey anti-goat and fluorescein-conjugated donkey anti-mouse antibody provided secondary detection. Synovial vascularity was quantified by fluorescent pixel area of synovial sections stained with anti-vWf, with computer analysis (IP Lab Spectrum software; Signal Ana-

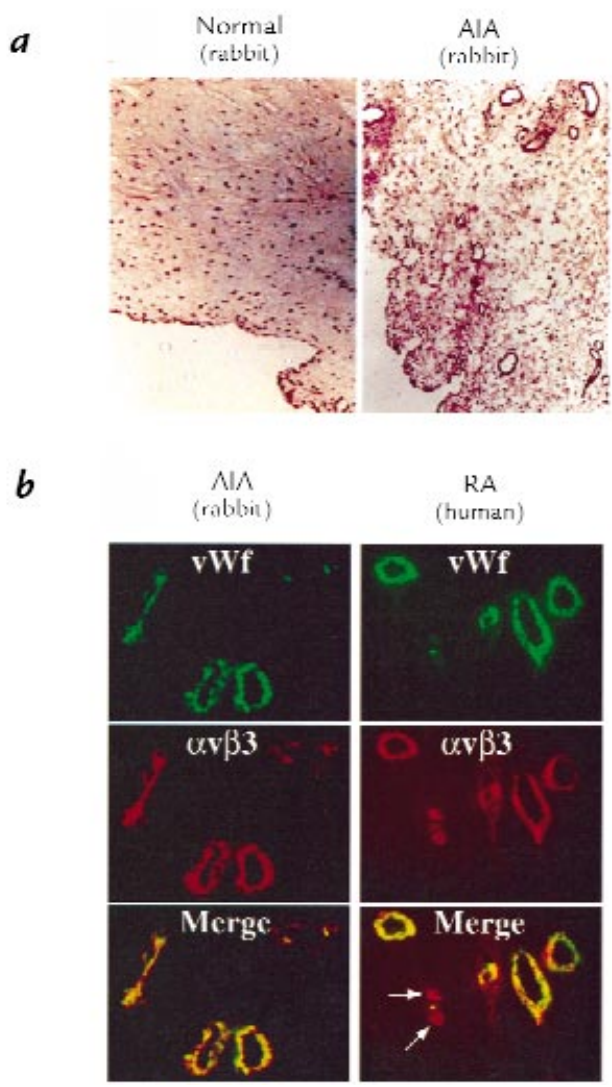

Figure 2

AIA and human RA exhibit $\alpha v \beta 3$ expression on angiogenic blood vessels. (a) Cryosections of infrapatellar tissue in AIA stained with H\&E demonstrate marked neovascularization, synovial hypertrophy, and dense synovial inflammatory infiltrate relative to synovium from nonarthritic tissue $(\times 100)$. (b) Immunofluorescent detection of blood vessels by vWf-specific antibody (green) and MAB LM609 directed to integrin $\alpha v \beta 3$ (red) demonstrates colocalization of $\alpha v \beta 3$ on the synovial endothelium (yellow merge) in rabbit AIA, as in human $\mathrm{RA}(\times 400)$. Note that microvascular angiogenic sprouts express only $\alpha v \beta 3$ (arrows). H\&E, hematoxylin and eosin; $M A B$, monoclonal antibody; $R A$, rheumatoid arthritis; $v W f$, von Willebrand factor. 


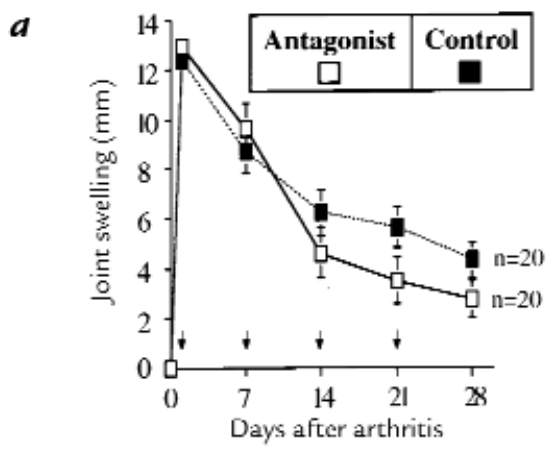

$\boldsymbol{b}$

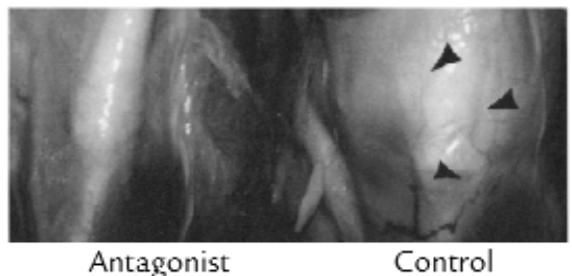

Figure 3

Joint swelling is reduced after $\alpha v \beta 3$ antagonist administration in AIA. (a) Rabbits with AIA were treated with bilateral intra-articular $\alpha v \beta 3$ antagonist (EMD 66203; $0.5 \mathrm{ml}, 2 \mathrm{mg} / \mathrm{ml}$ ) or control peptide (EMD 69601) beginning $24 \mathrm{~h}$ after arthritis onset, and weekly thereafter for 4 weeks (arrows). Joint swelling, defined as the increase in knee diameter from normal (nonarthritic), was significantly reduced by the $\alpha v \beta 3$ antagonist (days 14-28, $P<0.05$, ANOVA) compared with control peptide. (b) Periarticular vascularization (arrowheads) was prominent in control-treated AIA compared with antagonist-treated groups.

lytics Corp., Fairfax, Virginia, USA) of three 200× digital images per knee (CCD 1317 camera; Princeton Instruments Inc., Trenton, New Jersey, USA, and Axiovert 100 microscope; Carl Zeiss Inc., Thornwood, New York, USA. Angiogenic index was defined as [(test $v W f^{+}$area per field) - (nonarthritic vWf ${ }^{+}$area per field)] / (nonarthritic vWf ${ }^{+}$area per field). Synovial cell counts were obtained by automated computer counting of nuclei of digital images (three per knee, 400×) from hematoxylin and eosin (H\&E)-stained cryosections $(5 \mu \mathrm{m})$ of the infrapatellar fat-pad. Pannus formation was graded $0-5$ as described previously (19) $(0=$ normal to $5=$ pannus accompanied by cartilage erosion $)$. Erosions were assessed as present or absent by visual examination of femoral condyles. Apoptosis was detected using immunofluorescence detection of terminal deoxynucleotidyl transferase-mediated dUTP nick end-labeling (TUNEL) (ApopTag-Red; Oncor Inc., Gaithersburg, Maryland, USA), per supplier's protocol, on infrapatellar cryosections obtained from the right knee of each rabbit. Apoptotic vessels were scored as a percentage of total vessels observed (20 fields [400×] per specimen) by costaining for $\mathrm{vWf}$. Hematological parameters were assessed by Biomedical Testing Services (San Diego, California, USA).
Results of early treatment are pooled data from separate experiments expressed as mean \pm SE. Statistical evaluation was performed with ANOVA or Student's $t$ test (JMP IN software; Duxbury Press, San Francisco, California, USA), with $P<0.05$ considered significant.

Characterization of antagonist activity. Migration toward RA synovial fluid (1:10 in DMEM) in the presence or absence of peptide antagonists $(1 \mathrm{mg} / \mathrm{ml})$ was assessed in transwell assays, using purified radiolabeled AIA rabbit leukocytes as reported previously (20). The relative affinity of the individual antagonists for isolated integrins was determined via soluble ligandbinding studies, as published (21).

\section{Results}

The angiogenic growth factor $b F G F$ enhances arthritic disease. To assess the effect of increased angiogenesis on arthritis development, the proangiogenic cytokine bFGF was added to antigen during arthritis induction. Recent evidence has shown bFGF to be upregulated and localized to the pannus-cartilage interface in RA tissues (5). The inclusion of bFGF in this model resulted in more consistent and accelerated arthritis than with antigen alone, characterized by greater joint swelling (Fig. 1a), increased pannus formation (Fig. 1b), and more frequent erosive disease (Fig. 1c). These results demonstrate that bFGF, an angiogenic growth factor, can exacerbate arthritis.

Integrin $\alpha v \beta 3$ is selectively expressed on synovial blood vessels in AIA and in buman RA. The rabbit AIA model resembles human RA in several important aspects, including histopathology and its response to therapeutic agents $(18,22)$. Rabbit AIA displays marked neovascularization accompanied by synovial growth and subsynovial inflammatory infiltrate (Fig. 2a). Notably, $\alpha v \beta 3$ staining in AIA is restricted to synovial blood vessels (Fig. 2b), as in human RA, which is indicative of ongoing angiogenesis; blood vessels in sections from normal, nonarthritic synovium express little or no $\alpha v \beta 3$ (data not shown). The earliest invasive blood vessels, although positive for $\alpha v \beta 3$, do not stain for $\mathrm{vWf}$ (Fig $2 b$, arrows), reflecting the fact that vWf is a marker of differentiated endothelium (23). As vessels mature, $\alpha v \beta 3$ is lost (24). The hyperproliferative synovial lining and subsynovial tissue also contain numerous fibroblasts and macrophages (Fig. 2a) (1). In contrast to cutaneous wound fibroblasts, which express integrin $\alpha v \beta 3$ (25), little or no expression of this integrin was found on synovial fibroblasts (Fig. 2b), in agreement with previous studies $(14,15,26,27)$. With respect to synovial macrophages, $\alpha v \beta 3$ expression was found to be restricted to osteoclasts and was not present in other monocyte/macrophage-derived cells (28).

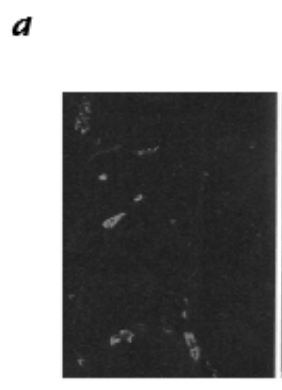

Arıt.agonist.

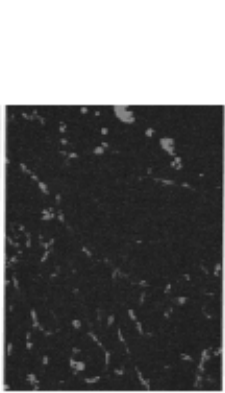

Control

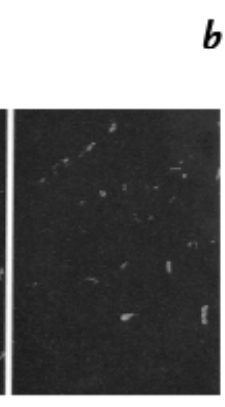

No arthritis

$\boldsymbol{b}$

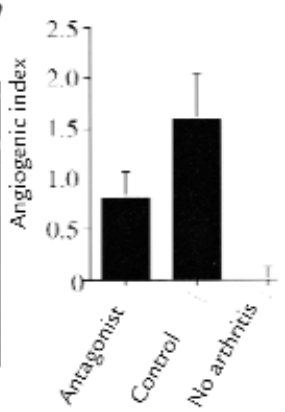

\section{Figure 4}

Synovial vascularity is decreased after $\alpha v \beta 3$ antagonist treatment. (a) Cryosections of the infrapatellar tissue obtained 28 days after arthritis onset in animals treated with the $\alpha v \beta 3$ antagonist or control peptide (days 1, 7, 14, and 21) were stained for vWf as a marker of blood vessels, detected with FITC-labeled secondary antibody, and were digitally imaged $(\times 200)$. $(\boldsymbol{b})$ The relative increase in area of fluorescent pixels per field vs. normal was computed to determine the angiogenic index as described in Methods. Synovial neovascularization was significantly inhibited by the $\alpha v \beta 3$ antagonist $(P<0.050$, Student's $t$ test). Data are expressed as mean $\pm \mathrm{SE}(n=20)$. 
Synovial neovascularization is inhibited by an $\alpha v \beta 3$ antagonist that targets angiogenic blood vessels. Blockade of integrin $\alpha v \beta 3$ by either antibody or peptide antagonists successfully inhibits neovascularization in cytokine and tumor models of angiogenesis in avian, murine, rabbit, and human tissues (11-13). To evaluate whether blockade of integrin $\alpha v \beta 3$ could impact arthritis-associated angiogenesis, rabbits with AIA were treated intra-articularly 24 hours after arthritis onset and weekly thereafter for four weeks with the $\alpha v \beta 3$ antagonist EMD 66203. This treatment resulted in a modest yet significant $(P<0.05)$ decrease in joint swelling compared with that in animals treated with the control peptide, EMD 69601 (Fig. 3a). Initial examination of knee joints revealed obvious periarticular vascularization in the control-treated animals (Fig. 3b). Cryosections of the infrapatellar fat-pad were examined with anti-vWf as a blood vessel marker. Tissues from rabbits treated with the $\alpha v \beta 3$ integrin antagonist displayed considerably fewer blood vessels than tissues from control-treated rabbits (Fig. 4a). Blinded computer analysis of digital images revealed a $48 \%$ decrease in synovial neovascularization in $\alpha v \beta 3$ antagonist-treated animals (Fig. $4 b)(P<0.01)$.

To determine whether the $\alpha v \beta 3$ antagonist was targeted to the vasculature in the arthritic synovium, we evaluated the distribution of a fluorescent-labeled form of this peptide, EMD 80838. Twenty-four hours after intra-articular injection, the fluorescent-conjugated $\alpha \mathrm{v} \beta 3$-binding peptide selectively localized to microvessels within the arthritic synovium, as determined by confocal microscopy (Fig. 5). These studies demonstrate that the $\alpha v \beta 3$ antagonist targets synovial blood vessels in this model and inhibits angiogenesis in the arthritic synovium.

Animals treated with the $\alpha v \beta 3$ antagonist show reduced synovial infiltrate. To evaluate whether the observed angiogenesis inhibition in $\alpha v \beta 3$ antagonist-treated animals was associated with a change in arthritis, several disease parameters were examined. Angiogenesis inhibition has been postulated to limit accessibility of the tissue to leukocytes (3); therefore, we first examined whether there was any decrease in the degree of synovial inflammatory infiltrate in animals treated with the antiangiogenic $\alpha v \beta 3$ antagonist. Histological examination of the infrapatellar
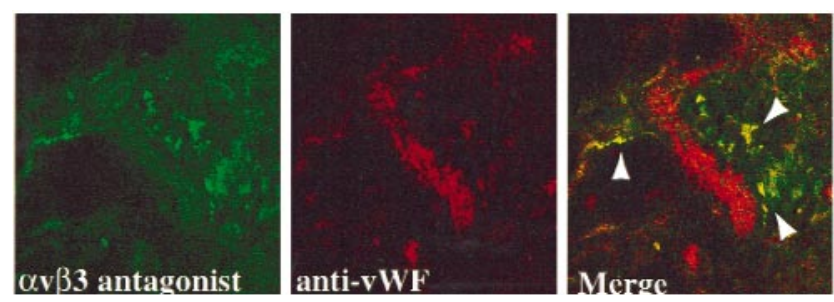

\section{Figure 5}

Fluorescent integrin antagonist colocalizes with synovial angiogenic microvessels. An FITC-conjugated $\alpha v \beta 3$ antagonist peptide, EMD 80838 (cyclic-Arg-Gly-Asp-D-Phe-Lys-[fluoresceincarboxylic acid]; $0.5 \mathrm{mg} / 500 \mu \mathrm{l}$ ), was injected intra-articularly in rabbits with AIA. After $24 \mathrm{~h}$, cryostat sections $(5 \mu \mathrm{m})$ of synovial tissue were examined by confocal microscopy for the presence of peptide (green). Blood vessels were identified by antisera to VWF as detected with TRITC-labeled secondary antibody $($ red $)$. Colocalization of $\alpha v \beta 3$ antagonist is observed on angiogenic microvessels after signal merge (yellow), but not with mature $\mathrm{VWF}^{+}$vessels $(\times 630)$. TRITC, tetrarhodamine isothiocyanate.

fat-pad revealed that treatment with the $\alpha v \beta 3$ antagonist resulted in significantly fewer cells infiltrating the synovium (Fig. 6a). Computerized cell counting of digital images of these sections demonstrated a 39\% reduction in synovial cellular infiltrate (Fig. $6 b)(P<0.05)$. Since only a small fraction of peripheral lymphocytes $(1 \%-2 \%)$ express integrin $\alpha v \beta 3$ (29), it is unlikely that the reduction in cellular infiltrate was due to leukocyte integrin inhibition. In fact, chemotaxis of AIA peripheral blood mononuclear cells (PBMCs) and polymorphonuclear neutrophils (PMNs) toward RA synovial fluid was not influenced by either the $\alpha v \beta 3$-directed peptide or the control peptide on collagen (Fig. 6c) or fibronectin (data not shown). No leukopenia or other sign of hematologic or marrow toxicity was observed after treatment in either group (Table 1). Immunohistochemical analysis revealed no difference in the degree of colocalization between apoptotic cells and leukocytes, as determined by TUNEL and KEN-11 (anti-LFA-1) costaining studies between control and $\alpha v \beta 3$ antagonist-treated rabbits (data not shown). Thus, the reduced synovial infiltrate observed in the $\alpha v \beta 3$ antagonist-treated AIA synovium does not appear to result from direct effects of this compound on leukocyte number or migration.

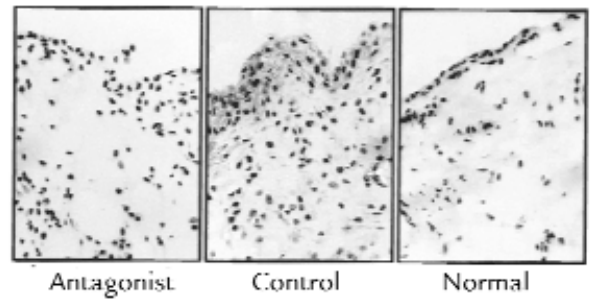

b

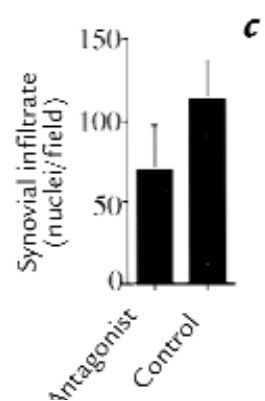

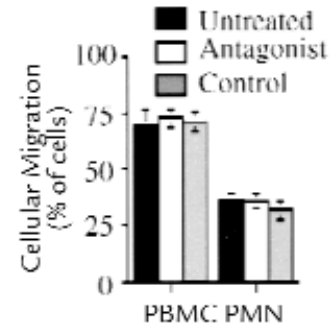

PBMC: PMN

\section{Figure 6}

Integrin $\alpha v \beta 3$ antagonist reduces synovial inflammatory infiltrate in AIA without impairing leukocyte migration. (a) H\&E stain of cryosections of the infrapatellar fat-pad 28 days after arthritis onset reveals a marked decrease in synovial cellular infiltrate in animals treated with the $\alpha v \beta 3$ antagonist (days 1, 7, 14, and 21) compared with control. (b) Digital assessment of nuclei present in each field (three per joint, $\times 400)$ demonstrates that $\alpha v \beta 3$ antagonist treatment resulted in a significant reduction in the cellular infiltrate $(P<0.05$, Student's $t$ test). Data are expressed as mean \pm SE ( $n=20)$. (c) In vitro chemotaxis on type II collagen toward synovial fluid by PBMCs or PMNs isolated from peripheral blood from rabbits with AIA. Data are expressed as mean \pm SE of triplicate determinations. No effect of the $\alpha v \beta 3$ antagonist on the migratory capacity of leukocytes was observed. PBMCs, peripheral blood mononuclear cells; PMNs, polymorphonuclear neutrophils. 
Table 1

No hematologic toxicity with $\alpha v \beta 3$ antagonist treatment

\begin{tabular}{lccc} 
Hematologic parameters & Pre-arthritis & \multicolumn{2}{c}{ Post-treatment } \\
& & $\alpha v \beta 3$ Antagonist & Control \\
WBC & $5.2 \pm 1.2 / \mathrm{nl}$ & $5.1 \pm 1.6$ & $4.0 \pm 0.6$ \\
\% PMNs & $52 \pm 11$ & $66 \pm 9$ & $62 \pm 1$ \\
$\%$ Lymphocytes & $40 \pm 10$ & $24 \pm 10$ & $26 \pm 5$ \\
RBC & $5.9 \pm 0.3 / \mathrm{pl}$ & $6.0 \pm 0.4$ & $6.4 \pm 0.4$ \\
Hemoglobin & $12.2 \pm 0.3 \mathrm{~g} / \mathrm{dl}$ & $12.6 \pm 0.4$ & $13.1 \pm 0.4$ \\
Platelets & $754 \pm 162 / \mathrm{nl}$ & $542 \pm 115$ & $486 \pm 79$
\end{tabular}

Complete blood count and manual differential were performed on blood samples obtained before arthritis induction, and again at time of harvest, in rabbits with AIA treated with four weekly bilateral intraarticular injections of the $\alpha v \beta 3$ antagonist (cyclic Arg-Gly-Asp-D-Phe-Val, EMD 66203) or control peptide (cyclic Arg-Ala-AspD-Phe-Val, EMD 69601) $(0.5 \mathrm{ml} / \mathrm{knee}, 2 \mathrm{mg} / \mathrm{ml})$. No lymphopenia or any other evidence of hematologic toxicity was observed with the $\alpha v \beta 3$ antagonist treatment. Data shown are mean $\pm \mathrm{SD}(n=4)$. AlA, antigen-induced arthritis; $P M N s$, polymorphonuclear neutrophils; $R B C$, erythrocyte count; $W B C$, white blood count.

The $\alpha v \beta 3$ antagonist protects against erosive disease. Since pannus formation is thought to initiate progression to erosive disease (1), the effect of the $\alpha v \beta 3$ antagonist on the development of pannus was examined. In control-treated animals, extensive pannus formation covering much of the femoral articular cartilage surface was observed, whereas treatment with the $\alpha v \beta 3$ antagonist resulted in significant reduction of pannus development $(P<0.05)$ (Fig. $7 a$ ). The $\alpha v \beta 3$ antagonist also significantly blocked the development of cartilage erosions. Gross erosions were present in $70 \%$ (14 of 20) of femoral condyles in controltreated AIA, yet only $20 \%$ (4 of 20) treated with the $\alpha v \beta 3$ antagonist exhibited erosions (Fig. $7 b)(P<0.01)$.

Vascular integrin $\alpha v \beta 3$ is the preferred target in AIA. Recent studies suggest that both integrin $\alpha \mathrm{v} \beta 3$ and integrin $\alpha v \beta 5$ can play a role in angiogenesis (30). Therefore, we compared the antiarthritic effects of the cyclic peptide with $\alpha v \beta 3$ selectivity, EMD 66203, with that of a peptide with higher $\alpha v \beta 5$-binding activity, EMD 85189. Notably, $\alpha v \beta 5$ is also highly expressed on synovial fibroblasts and macrophages $(14,27)$. The peptide antagonist with greatest selectivity for $\alpha v \beta 3$ demonstrated strongest inhibition of pannus development and erosive disease (Table 2 ), suggesting that $\alpha v \beta 3$ was the primary therapeutic target in this model.
Chronic arthritis is ameliorated by the $\alpha v \beta 3$ antagonist. We extended these studies to evaluate the effect of $\alpha v \beta 3$ blockade on well-established arthritis. AIA was allowed to progress for two weeks into the chronic phase (18) before the initiation of treatment. Despite the delay, joint swelling was significantly decreased by the $\alpha v \beta 3$ antagonist (Fig. 8a). In this chronic model, treatment with the antagonist produced a significant antiangiogenic effect, with a $64 \%$ reduction in the angiogenic index compared with untreated AIA $(P<0.05)$ (Fig. $8 b)$, and blockade of $\alpha v \beta 3$ was again associated with a marked reduction in the synovial cell infiltrate $(P<$ 0.05) (Fig. 8c). As observed previously with early administration during acute disease, delayed treatment with the $\alpha v \beta 3$ antagonist decreased pannus formation and reduced the incidence of erosive disease compared with controls (Fig. 8, $d$ and e). From these results, we conclude that angiogenesis contributes to disease severity as well as chronicity, and that an antagonist of integrin $\alpha v \beta 3$ is an effective antiarthritic agent in well-established disease.

Synovial vascular apoptosis is associated with $\alpha v \beta 3$ antagonist treatment. Previous studies have demonstrated that antagonists of integrin $\alpha \mathrm{v} \beta 3$ induce apoptosis of angiogenic blood vessels (11-13). Therefore, we examined synovial tissues from the chronic arthritis model for the presence of apoptotic cells after treatment with the $\alpha v \beta 3$ antagonist. Immunohistochemical detection of apoptosis within AIA synovium demonstrated increased (2.5-fold) TUNEL-positive vascular cells in the $\alpha v \beta 3$ antagonist-treated animals $(P<0.01)$ (Fig. 9). These observations suggest that $\alpha v \beta 3$ antagonists may inhibit angiogenesis in the arthritic synovium by inducing apoptosis of angiogenic blood vessels.

\section{Discussion}

RA has been classified as an angiogenesis-associated disease, based on the finding of extensive neovascularization within the inflamed synovium (3). Clearly, inflammatory cytokines with angiogenic activity can induce or potentiate synovitis $(31,32)$. However, it has not been determined whether angiogenesis directly contributes to the arthritic process or whether it is merely a consequence of ongoing inflammation. Evidence is provided in this report that angiogenesis contributes to arthritic disease.

Table 2

Integrin $\alpha v \beta 3$ selective antagonist ameliorates AIA

\begin{tabular}{|c|c|c|c|c|}
\hline Antagonist IC $\mathrm{C}_{50}$ vs. & $\begin{array}{c}\alpha v \beta 3 \text { antagonist } \\
\text { (cRGDfV) }\end{array}$ & $\begin{array}{l}\alpha v \beta 3 / \beta 5 \text { antagonist } \\
\text { (cRGDf } N \text {-methyl]-V) }\end{array}$ & $\begin{array}{c}\text { Control } \\
\text { (cR- } \beta A-D f V)\end{array}$ & No treatment \\
\hline Integrin $\alpha v \beta 3$ & $4.8 \mathrm{nM}$ & $2.3 \mathrm{nM}$ & $>>1 \mu \mathrm{M}$ & $\mathrm{n} / \mathrm{a}$ \\
\hline Integrin $\alpha v \beta 5$ & $450 \mathrm{nM}$ & $37 \mathrm{nM}$ & $>>1 \mu \mathrm{M}$ & $\mathrm{n} / \mathrm{a}$ \\
\hline \multicolumn{5}{|l|}{ Antagonist effect on AIA } \\
\hline Swelling (\% increase) & $39.5 \pm 10$ & $40.5 \pm 14$ & $57.4 \pm 10$ & $56.8 \pm 25$ \\
\hline Pannus (0-5) & $1.4 \pm 0.6$ & $2.9 \pm 0.7$ & $3.8 \pm 0.6$ & $3.0 \pm 1.2$ \\
\hline Erosions (\% incidence) & $1 / 8(12.5 \%)$ & $3 / 8(37.5 \%)$ & $5 / 8(62.5 \%)$ & $2 / 4(50 \%)$ \\
\hline
\end{tabular}

Determinations of peptide antagonist $I C_{50}$ on isolated integrins were performed as described previously (24). Rabbits with bilateral AIA were treated with intra-articular injection of peptides $(2 \mathrm{mg} / \mathrm{ml}, 500 \mu \mathrm{l})$ beginning $24 \mathrm{~h}$ after arthritis induction and on days 7, 14, and 21 . After 4 weeks, joint swelling, pannus formation, and the presence of macroscopic erosions were evaluated as detailed in Methods. All data are shown as mean $\pm \operatorname{SE}(n=8$ peptides; $n=4$ untreated $)$. 

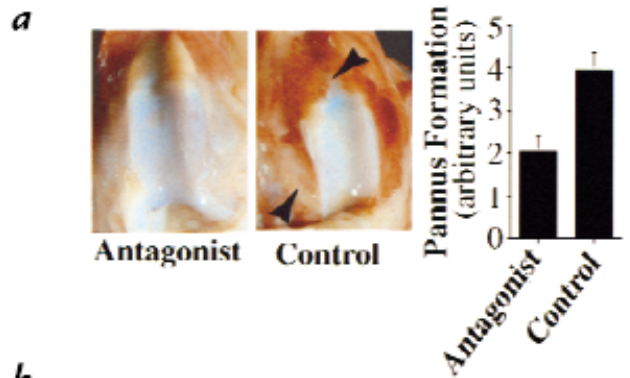

$b$
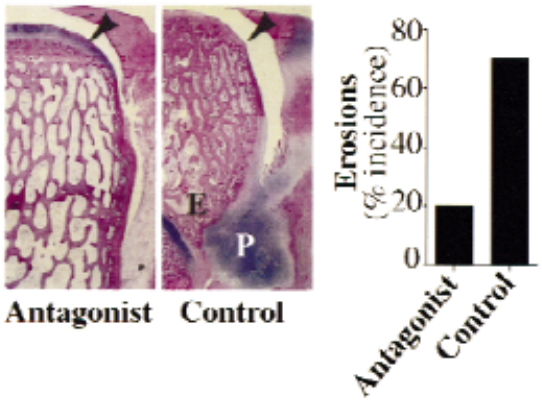

Figure 7

Blockade of integrin $\alpha v \beta 3$ decreases pannus formation and cartilage erosions. (a) Pannus development (arrowheads) was graded on a relative scale $0-5(0=$ normal to $5=$ macroscopic erosion $)$. Treatment with the $\alpha v \beta 3$ antagonist significantly decreased pannus formation $(P<0.05$, Student's $t$ test) relative to control peptide. (b) Frontal sections of decalcified femoral condyle stained by $\mathrm{H} \& \mathrm{E}$ illustrate the protective effect of $\alpha v \beta 3$ antagonist treatment on a representative sample of erosive disease $(\times 10)$. ( $P$ indicates pannus, $E$ indicates erosion, and the arrowhead indicates preservation of the articular cartilage in the antagonist-treated group.) Macroscopic cartilage erosions were decreased with $\alpha v \beta 3$ antagonist treatment $(P<0.01$, Student's $t$ test). All data are expressed as mean $\pm \operatorname{SE}(n=20)$.
Addition of the proangiogenic cytokine bFGF to AIA accelerated and exacerbated arthritis. The inclusion of bFGF in this model was based on the facts that bFGF does not possess the inflammatory properties associated with other angiogenic factors (e.g., interleukin-8 [31] andtumor necrosis factor- $\alpha$ [32]), bFGF is upregulated and localized to the pannus-cartilage interface in RA tissues (5), and synovial levels of bFGF in human RA correlate with disease severity (33). In fact, the addition of bFGF to AIA produced an accelerated and more consistent arthritis compared with arthritis induced with antigen alone (Fig. 1), without altering the existing expression and selective distribution of integrin $\alpha v \beta 3$, as assessed by staining with LM609 (data not shown).

Not only did bFGF accelerate arthritis in this model, but a peptide antagonist selective for integrin $\alpha v \beta 3$ inhibited angiogenesis and reduced the severity of arthritis. Joint swelling, synovial infiltrate, pannus formation, and the incidence of cartilage erosions were all significantly reduced in animals treated early with this integrin antagonist. Inhibition of neovascularization, as demonstrated in this model, may influence many parameters of disease, including inflammation, protease production, and tissue metabolism (3). No evidence for a direct role of the $\alpha v \beta 3$ antagonist in impairing peripheral leukocyte migration was detected, nor was acute inflammation affected in antagonist-treated animals upon rechallenge with antigen (data not shown).

Integrin $\alpha v \beta 3$ was selectively localized to synovial blood vessels in human RA and rabbit AIA, as determined by costaining with LM609 and anti-vWf (Fig. 2b). The appearance of $\alpha v \beta 3$-positive/vWf-negative vessels reflects the fact that $\mathrm{vWf}$ is a marker of more mature vessels (23), whereas $\alpha v \beta 3$ is present on very early neovessels (34). In addition, the $\alpha v \beta 3$ antagonist localized to microvessels 24 hours after its intra-articular administration in the arthritic synovium, supporting previous findings that this integrin is found primarily on angiogenic blood vessels $(11,34)$. $\boldsymbol{a}$
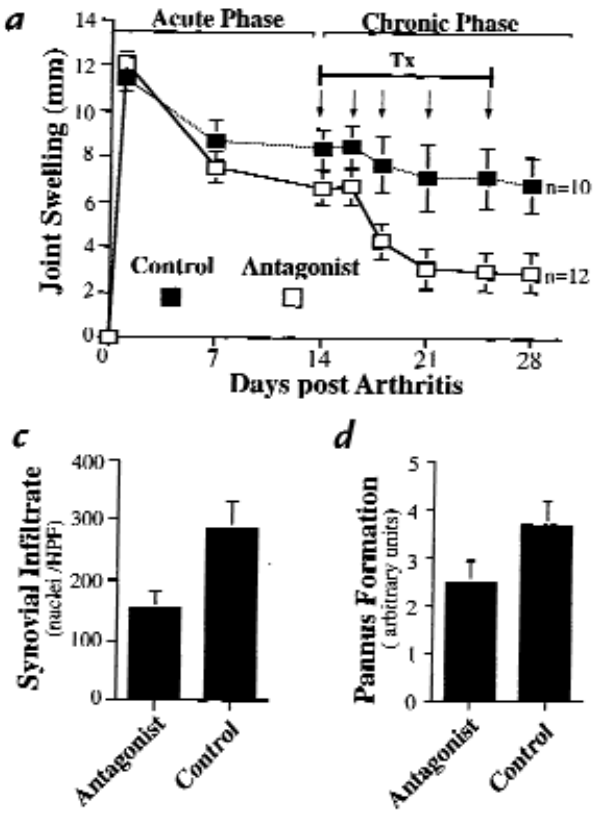

$d$

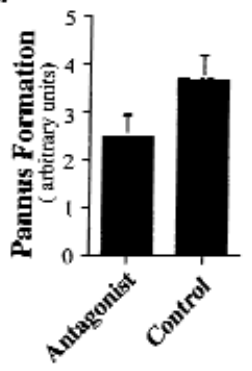

b

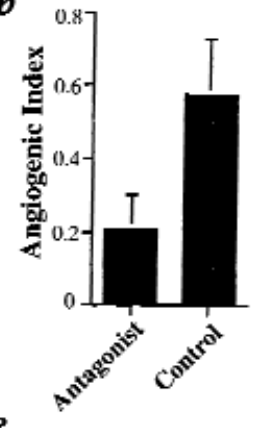

$e$

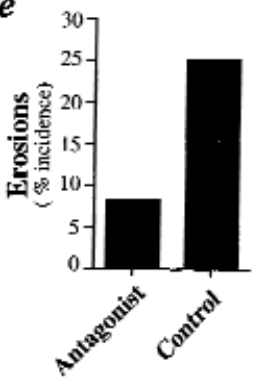

\section{Figure 8}

Chronic arthritis is ameliorated by an $\alpha v \beta 3$ antagonist. (a) Administration of the antagonist (arrows) beginning 2 weeks after arthritis onset resulted in decreased joint swelling compared with controls (untreated) $(P<0.05$, ANOVA). (b) Angiogenesis, as depicted by the angiogenic index (see Methods), was inhibited by $\alpha v \beta 3$ antagonist treatment $(P<0.05$, Student's $t$ test). (c) Fewer infiltrating cells were observed in the synovium of $\alpha v \beta 3$ antagonist-treated animals $(P<0.05$, Student's $t$ test), as assessed by digital computerized counting of nuclei. (d) Pannus was assessed as described in Methods. (e) A decrease in both pannus formation and cartilage erosion was observed in antagonist-treated animals relative to control. Data are expressed as mean \pm SE $(n=12$, antagonist; $n=10$, control). HPF, highpower field; $T x$, treatment. 
During maturation and differentiation, monocytes/macrophages may express $\alpha_{\mathrm{v}}$ integrins $(28,35,36)$, and in fact, antagonists of $\alpha v \beta 3$ can influence macrophage-derived osteoclast function (37), which may contribute to the observed decrease in bone erosions after $\alpha v \beta 3$ antagonist treatment. However, functionally mature $\left(\alpha v \beta 3^{+}\right)$osteoclasts are rarely found in the synovium (38), and it is uncertain that the short-term, intermittent dosing regimen of the $\alpha v \beta 3$ antagonist used in this study would be sufficient to impact ongoing osteoclast function. Although the $\alpha v \beta 3$-selective cyclic peptide antagonist can impact other $\alpha v$ integrins, it is ineffective against $\beta 1$ integrin-mediated adhesion (data not shown). Antagonism of integrin $\beta 1$ with small Arg-Gly-Asp -containing linear peptide fragments of fibronectin induces collagenase expression in synovial fibroblasts, and administration of these peptides may thereby actually increase disease (39). Although $\alpha v \beta 3$ is present in certain fibroblast populations (25), it was not detected in fibroblasts from synovial tissues in patients with RA $(14,26,27)$.

The integrin antagonist used, although selective for integrin $\alpha v \beta 3$, exhibits some activity against the closely related integrin $\alpha v \beta 5$ (Table 2). Antagonism of integrin $\alpha v \beta 5$ is sufficient to block angiogenesis induced by some cytokines (30) and thus may have contributed to the reduced synovial vascularization observed. However, administration of an antagonist with a higher affinity for integrin $\alpha v \beta 5$, which is also expressed on synovial fibroblasts and macrophages $(14,27)$, resulted in only minor improvements in arthritis severity (Table 2), suggesting that vascular integrin $\alpha v \beta 3$ is the preferred target in inflammatory arthritis.

The results presented in this report provide evidence for a central pathogenic contribution of angiogenic blood vessels to both the induction and maintenance of arthritic disease. Increased neovascularization was clearly and consistently associated with more severe arthritis. Selective $\alpha v \beta 3$ blockade effectively inhibited synovial angiogenesis and provided significant protection against the development of cellular infiltrate, pannus formation, and cartilage erosions. In view of the rather selective distribution of integrin $\alpha \mathrm{v} \beta 3$ on blood vessels in the arthritic synovium (Fig. 2b) (14, 15, 26-28), the quantitative increase in endothelial apoptosis after $\alpha v \beta 3$ antagonist treatment, and the decreased vascularity observed, angiogenesis inhibition is a likely mechanism by which $\alpha v \beta 3$ antagonists ameliorate arthritis. The fact that an $\alpha v \beta 3$ antagonist was effective when administered in the chronic AIA model is relevant to future clinical applications. The expression of integrin $\alpha v \beta 3$ on synovial blood vessels in human RA presents an opportunity to pursue $\alpha v \beta 3$ integrin-directed antiangiogenic therapy for the treatment of RA.

\section{Acknowledgments}

The authors thank Melinda Storgard for excellent technical assistance. This work was supported in part by grants from the Department of Academic Affairs of Scripps Clinic and the Jeanette Hennings Foundation. C.M. Storgard is a recipient of an Arthritis Foundation Postdoctoral Fellowship. D.G. Stupack is a recipient of a Canadian Arthritis Society Fellowship and a Joseph Drown Foundation Fellowship. D.A. Cheresh was supported by National Institutes of Health grants CA-50286, CA45726, and HL-54444. This is publication no. 11520-IMM from the Scripps Research Institute.
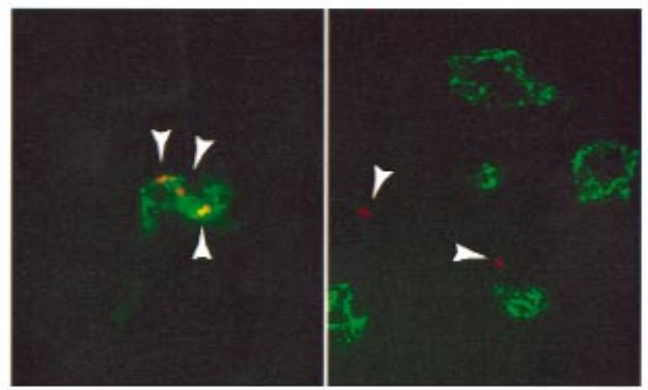

$b$

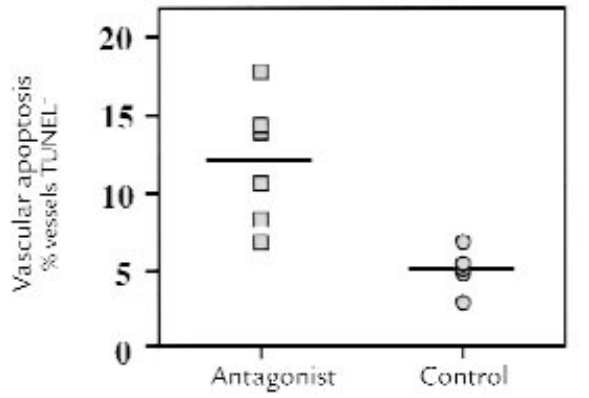

Figure 9

Vascular cell apoptosis is associated with $\alpha v \beta 3$ antagonist treatment. (a)Synovium from rabbits in the chronic AIA model were stained with TUNEL immunostaining (red) as an indicator of apoptosis, (arrowheads)and anti-vWF (green) as a marker of blood vessels ( $\times 400)$. (b) Apoptosis associated with blood vessels was detected and quantified blindly as percent blood vessels containing apoptotic cells in 20 fields $(\times 400)$ per specimen. Apoptosis was increased specifically in the vasculature of arthritic synovium after $\alpha v \beta 3$ antagonist treatment $(P<0.01$, Student's $t$ test). TUNEL, terminal deoxynucleotidyl transferase-mediated dUTP nick end-labeling.

1. Zvaifler, N.J., and Firestein, G.S. 1994. Pannus and pannocytes: alternative models of joint destruction in rheumatoid arthritis. Arthritis Rheum. 37:783-789.

2. Kimball, E.S., and Gross, J.L. 1991. Angiogenesis in pannus formation. Agents Actions. 34:329-331.

3. Colville-Nash, P.R., and Scott, D.L. 1992. Angiogenesis and rheumatoid arthritis: pathogenic and therapeutic implications. Ann. Rheum. Dis. 51:919-925.

4. Paleolog, E. 1997. Target effector role of vascular endothelium in the inflammatory response: insights from the clinical trial of anti-TNF alpha antibody in rheumatoid arthritis. Mol. Pathol. 50:225-233.

5. Qu, Z., et al. 1995. Expression of basic fibroblast growth factor in synovial tissue from patients with rheumatoid arthritis and degenerative joint disease. Lab. Invest. 73:339-346.

6. Koch, A.E. 1998. Review: angiogenesis: implications for rheumatoid arthritis. Arthritis Rheum. 4:951-962.

7. Matsubara, T., and Ziff, M. 1987. Inhibition of endothelial cell proliferation by gold compounds. J. Clin. Invest. 79:1440-1446.

8. Potvin, F., Petitclerc, E., Marceau, F., and Poubelle, P.E. 1997. Mechanisms of action of antimalarials in inflammation: induction of apoptosis in human endothelial cells. J. Immunol. 158:1872-1879.

9. Hirata, S., Matsubara, T., Saura, R., Tateishi, H., and Hirohata, K. 1989. Inhibition of in vitro vascular endothelial cell proliferation and in vivo neovascularization by low dose methotrexate. Arthritis Rheum. 32:1065-1073.

10. Peacock, D.J., Banquerigo, M.L., and Brahn, E. 1995. A novel angiogenesis inhibitor suppresses rat adjuvant arthritis. Cell. Immunol. 160:178-184.

11. Brooks, P.C., Clark, R.A., and Cheresh, D.A. 1994. Requirement of vascular integrin $\alpha v \beta 3$ for angiogenesis. Science. 264:569-571.

12. Brooks, P.C., et al. 1995. Anti-integrin $\alpha v \beta 3$ blocks human breast cancer growth and angiogenesis in human skin. J. Clin. Invest. 96:1815-1822.

13. Brooks, P.C., et al. 1994. Integrin $\alpha v \beta 3$ antagonists promote tumor regression by inducing apoptosis of angiogenic blood vessels. Cell. 79:1157-1164. 
14. Johnson, B.A., Haines, G.K., Harlow, L.A., and Koch, A.E. 1993. Adhesion molecule expression in human synovial tissue. Arthritis Rheum. 36:137-146.

15. Walsh, D.A., Wade, M., Mapp, P.I., and Blake, D.R. 1998. Focally regulated endothelial proliferation and cell death in human synovium. Am. J. Pathol. 152:691-702.

16. Cheresh, D.A., and Spiro, R.C. 1987. Biosynthetic and functional properties of Arg-Gly-Asp-directed adhesion receptor involved in human melanoma cell attachment to vitronectin, fibrinogen, and von Willebrand factor. J. Biol. Chem. 262:17703-17711.

17. Aumailley, M., et al. 1991. Arg-Gly-Asp constrained within cyclic pentapeptides: strong and selective inhibitors of cell adhesion to vitronectin and laminin fragment P1. FEBS Lett. 291:50-54.

18. Jason, H.E. 1988. Chronic arthritis in rabbits. Methods Enzymol. 162:379-385.

19. Maksymowych, W.P., et al. 1996. Amelioration of antigen induced arthritis in rabbits treated with a secreted viral serine proteinase inhibitor. $J$. Rheumatol. 23:878-882.

20. Taub, R.N. 1974. Effects of concanavalin A on the migration of radioactively labeled lymphoid cells. Cell. Immunol. 12:263-270.

21. Haubner, R., et al. 1996. Cyclic RGD peptides containing $\beta$-turn mimetics. J. Am. Chem. Soc. 118:7881-7891.

22. Hunneyball, I.M. 1984. Use of experimental arthritis in the rabbit for the development of antiarthritic drugs. Adv. Inflamm. Res. 7:249-262.

23. Han, R.N., Tanswell, A.K., and Post, M. 1992. Ontogeny of reactivity to endothelial cell markers during development of the embryonic and fetal rat lung. Histol. Histopathol. 7:591-597.

24. Clark, R.A., Tonnesen, M.G., Gailit, J., and Cheresh, D.A. 1996. Transient functional expression of $\alpha v \beta 3$ on vascular cells during wound repair. Am. J. Pathol. 148:1407-1421.

25. Gailit, J., and Clark, R.A. 1996. Studies in vitro on the role of alpha $v$ and beta 1 integrins in the adhesion of human dermal fibroblasts to provisional matrix proteins fibronectin, vitronectin, and fibrinogen. J. Invest. Dermatol. 106:102-108.

26. Rinaldi, N., et al. 1997. Differential expression and functional behavior of the alpha $v$ and beta 3 integrin subunits in cytokine stimulated fibroblast-like cells derived from synovial tissue of rheumatoid arthritis and osteoarthritis in vitro. Ann. Rheum. Dis. 56:729-736.
27. Nikkari, L., et al. 1995. Localization of the alpha v subfamily of integrins and their putative ligands in synovial lining cell layer. J. Rheumatol. 22:16-23.

28. Zambonin, Z.A., Teti, A., Gaboli, M., and Marichisio, P.C. 1989. Beta 3 subunit of vitronectin receptor is present in osteoclast adhesion structures and not in other monocyte-macrophage derived cells. Connect. Tissue Res. 20:143-149.

29. Hemler, M.E. 1988. Adhesive protein receptors on hematopoietic cells. Immunol. Today. 9:109-113.

30. Friedlander, M., et al. 1995. Two pathways of angiogenesis defined by distinct $\alpha_{v}$ integrins. Science. 250:1500-1502.

31. Endo, H., et al. 1994. Experimental arthritis induced by continuous infusion of IL-8 into rabbit knee joints. Clin. Exp. Immunol. 96:31-35.

32. Lewthwaite, J., et al. 1995. Role of TNF alpha in the induction of antigen induced arthritis in the rabbit and the anti-arthritic effect of species specific TNF alpha neutralizing monoclonal antibodies. Ann. Rheum. Dis. 54:366-374.

33. Nakashima, M., et al. 1994. Expression of basic fibroblast growth factor in synovial tissues from patients with rheumatoid arthritis: detection by immunohistological staining and in situ hybridisation. Ann. Rheum. Dis. 53:45-50.

34. Drake, C.J., Cheresh, D.A., and Little, C.D. 1995. An antagonist of integrin $\alpha v \beta 3$ prevents maturation of blood vessels during embryonic neovascularization. J. Cell Sci. 108:2655-2661.

35. De Nichilo, M.O., and Yamada, K.M. 1996. Integrin $\alpha v \beta 5$-dependent serine phosphorylation of paxillin in cultured human macrophages adherent to vitronectin. J. Biol. Chem. 271:11016-11022.

36. Andreesen, R., et al. 1990. Surface phenotype analysis of human monocyte to macrophage maturation. J. Lenkoc. Biol. 47:490-497.

37. Engleman, V.W., et al. 1997. A peptidomimetic antagonist of the $\alpha v \beta 3$ integrin inhibits bone resorption in vitro and prevents osteoporosis in vivo. J. Clin. Invest. 99:2284-2292.

38. Ashton, B.A., Ashton, I.K., Marshall, M.J., and Butler, R.C. 1993. Localization of vitronectin receptor immunoreactivity and tartrate resistant acid phosphatase activity in synovium from patients with inflammatory or degenerative arthritis. Ann. Rheum. Dis. 52:133-137.

39. Homandber, G.A., Meyers, R., and Williams, J.M. 1993. Intraarticular injection of fibronectin fragments causes severe depletion of cartilage proteoglycans in vivo. J. Rheumatol. 20:1378-1382. 